\title{
A New Frequency Dependent Approach to Model Validation
}

\author{
Pedro Balaguer and Ramon Vilanova \\ Autonomous University of Barcelona \\ Spain
}

\section{Introduction}

In order to have confidence in a model it is necessary to validate it. Different model validation approaches exist. Their difference is based upon the assumptions about the plant and models. Classical validation methods, based on classical model identification (Ljung, 1994; Soderstrom and Stoica, 1989) rely on statistical uncertainty assumptions due to stochastic noise only. On the other hand, control oriented identification methods (Chen and $\mathrm{Gu}, 200)$ (i.e. $\mathcal{H}_{\infty}$ identification, stochastic embedding, set membership identification, etc.) lead to validation assumptions based on bounded noise and bounded model undermodelling. However in both cases the output of the validation process is just a "validated/invalidated" result.

Regarding classical validation methods, the problem of assessing the validity of an identified model has been traditionally linked with the problem of model order selection. The classical model validation literature (Ljung, 1994; Soderstrom and Stoica, 1989) has approached the problem in two ways:

- use of plots and common sense.

- use of statistical tests on the residuals $\xi(t)$ (i.e. the difference between the real output and the model output $\xi(t)=y(t)-\hat{y}(t))$.

The first approach is basically based on the comparison of experimental data with the model output. If both are similar then the model can be considered a good one. However there are two unavoidable reasons that prevent the model output to fit data perfectly: the modelling errors and perturbations. The second approach is to apply a hypothesis test over the residual $\xi(t)$, which follows from the assumptions on the disturbance. The more common assumptions over the residual are (Soderstrom and Stoica, 1989):

- $\mathrm{h} 1: \xi(t)$ is a zero mean white noise.

- $\mathrm{h} 2: \xi(t)$ has a symmetric distribution.

- $\mathrm{h} 3: \xi(t)$ is independent of past inputs (i.e. $E[\xi(t) u(\tau)]=0, t>\tau)$.

- $\mathrm{h} 4$ : $\xi(t)$ is independent of all inputs (i.e. $E[\xi(t) u(\tau)]=0, \forall t, \tau)$. 
The above assumptions lead to check two main residual properties, the whiteness of the residuals (i.e. h1, h2) and the cross-correlation between residuals $\xi(t)$ and control actions $u(t)$ (i.e. h3, h4).

Hence classical validation tests can be classified as follows

1. Whiteness Test.

- $\quad$ Autocorrelation test (Assumption h1).

- $\quad$ Testing changes of sign (Assumptions h1 and h2).

2. Independence between residuals and inputs.

- Cross-correlation test of past inputs (Assumption h3 or Assumptions h3 and h1).

- Cross-correlation test of all inputs (Assumption h4 or Assumptions h4 and h1).

The rationale of the tests is to detect causes of variation on the residual distinct than the ones assumed. For example if the residual is assumed to be white noise and the test shows that the whiteness statistical hypothesis is violated then we assume that there is a distinct cause producing the mismatch (i.e. model error).

The result of the statistical tests above reviewed is a binary one. In fact the test either validates or invalidates the model. No further information is provided by the test. As a result, two important drawbacks are

1. There is no information on important model aspects such as (Balaguer and Vilanova, 2006a):

- The reasons why the model is invalidate.

- How to improve the model.

- The model usefulness degree.

2. In control design, undermodelling (i.e. use of low order models) is normally present (Balaguer and Vilanova, 2006b). In fact as stated in (Ljung, 1994) "For such a model (a model simpler that the one that minimizes the total error) typically the bias error is the dominating contribution to the total error. Consequently, such models would normally be falsified during model validation".

Thus, as a conclusion, although the theory of classical validation methods is well developed and plenty of successful applications it has limitations when a more informative validation procedure is required.

In this chapter we present a new view to the model validation procedure. The main characteristic of the presented approach is that the validation is frequency dependent. As a result the validation procedure is more informative as:

1. The same model can result validated for a frequency range and invalidated for a distinct frequency band. Thus the result of the validation procedure is no longer binary but frequency dependent.

2. The algorithm provides the frequency range for which the model is validated.

3. The validation procedure is suited to be used with residuals generate in a control oriented fashion (i.e. in closed loop).

The application areas of this new, more informative, validation procedure are wide. The applicability potential hinges on the frequency dependence of the validation result. In particular we focus on the application for model validation on Iterative Identification and Control Design Schemes. In Iterative Identification and Control Design Schemes (Albertos and Sala, 2002), a new model is identified at each iteration. Thus it is necessary to validate the new model. Moreover these iterative schemes have some requirements, such as input 
experiment design, model order selection, etc. The proposed frequency dependent algorithm gives information in order to i) select controller bandwidth, ii) select model order and iii) design the input experiment for the next iteration.

A distinct application area of the algorithm presented here is the validation of controllers. The objective is to validate the behaviour of a possibly low order controller (e.g. PID) against a more complex controller (e.g. optimal controller). The procedure presented here can be applied to ascertain for what frequency ranges both controllers are behaving in an equivalent manner. Moreover the validation procedure also suggests a tuning method by means of minimizing the residual generated by the comparison of both controllers. First results can be found in (Balaguer et al., 2008).

Summing up, in this chapter we present a new model validation algorithm in which the validation result is no longer "validated/invalidated" but frequency dependent. Thus the validation result is more informative, providing new features of applicability in several areas of control theory.

The chapter contributions are organized as follows

1. A new procedure for model validation in the frequency domain is presented (Section 2 ). This procedure permits to validate or invalidate models over certain frequency ranges. The procedure is the translation of a time domain residual whiteness test to a frequency dependent residual whiteness test. The counterpart on the frequency domain of a time domain whiteness test is established. This leads to stating the statistical properties of each spectrum frequency component if the time domain transformed signal is white noise. It is shown that the normalized spectrum is a random variable with a $\chi^{2}$ distribution of 2 degrees of freedom (Theorem 2 ). The validation/invalidation step is based on a hypothesis test applied to each frequency component. This determines if certain frequency components have an unusual content that discards the model validity for this frequency value. The acceptance/rejection decision of the frequency component validity comes with a probability measure.

2. The intended use of the model (e.g. open loop or closed loop) is taken into account by considering the structure from which the residuals are generated. In Section 3 a study of the residuals information content and its statistical properties for different structures (e.g. open loop Vs closed loop) is conducted. As the validation procedure is based on a whiteness test, the residual should be white noise whenever the model fits accurately the plant. This however is not the general case when residuals coming from closed loop comparisons are used. In order to overcome this difficulty, a novel structure is proposed in Section 3.3 which is able to provide white residuals in closed loop.

3. The application of the presented validation procedure on Iterative Identification and Control Design Schemes is discussed in Section 4. It is shown that the validation procedure shifts from a classical static test (i.e. validation/invalidation) to a dynamic one which gives frequency domain information useful for improvement of identification and control design on iterative schemes.

4. The chapter ends in Section 5 stating the conclusions and the possible extensions of the frequency dependent validation algorithm. 


\section{Frequency Dependent Model Validation}

The main objective of the methodology presented is to validate a model on the frequency domain. To this end a time domain validation procedure based on testing the residual whiteness is modified to achieve the pursued objectives. The idea is as follows. It is assumed that if the residual is white noise the model is validated because the residual contains no further useful information that could be used to improve the model accuracy. This test is usually performed in the time domain by studying the residual autocorrelation, the number of sign changes, etc (Ljung, 1994).

We translate the time domain residual to the frequency domain by its discrete Fourier transform. Moreover, the statistical properties of the spectrum of a white noise signal are calculated. The objective is to test if the spectrum calculated from the residual has properties of white noise. As a result, one unique test in the time domain has been translated to $\mathrm{N}$ different tests in the frequency domain. We check if the $k^{\text {th }}$ frequency component of the spectrum has the properties of a typical frequency component of a white noise. In the affirmative case we have no reason to believe that the model is invalid on that frequency component. On the other hand, if there are certain frequency components that clearly do not behave accordingly with the statistical properties of white noise then it is likely that at this frequency range there is an important mismatch between the model and the plant. As a result the model is invalid for that frequency range.

\subsection{Whiteness Test on the Frequency Domain}

In this section the statistical time domain properties of a white noise are translated to the frequency domain. This is accomplished by means of two theorems. The first one is an intermediate result that is used by the second one which describes the frequency domain distribution of the spectrum of a white noise.

\section{Theorem 1}

Let $\xi(n)$ be a sequence of independent identically distributed (IID) samples of normal distribution $N\left(\mu_{\xi}, \sigma_{\xi}^{2}\right)$. If we express the discrete Fourier transform by its real and imaginary part, that is

$$
\xi_{k}=R_{k}+j I_{k}=\frac{1}{N} \sum_{n=0}^{N-1} \xi(n) e^{-j \Omega_{0} k n}
$$

then the real part $R_{k}$ is a random variable normally distributed $R_{k} \in N\left(\mu_{R_{k}}, \sigma_{R_{k}}^{2}\right)$ with mean $\mu_{R_{k}}$ and variance $\sigma_{R_{k}}^{2}$ given by

$$
\begin{gathered}
\mu_{R_{k}}=\mu_{\xi} \frac{1}{N} \sum_{n=0}^{N-1} \operatorname{Cos}\left(\Omega_{0} k n\right) \\
\sigma_{R_{k}}^{2}=\sigma_{\xi}^{2} \frac{1}{N^{2}} \sum_{n=0}^{N-1} \operatorname{Cos}^{2}\left(\Omega_{0} k n\right)
\end{gathered}
$$


Similarly the Imaginary part $I_{k}$ is a random variable normally distributed $I_{k} \in N\left(\mu_{I_{k}}, \sigma_{I_{k}}^{2}\right)$ with mean $\mu_{I_{k}}$ and variance $\sigma_{I_{k}}^{2}$ given by

$$
\begin{gathered}
\mu_{I_{k}}=\mu_{\xi} \frac{1}{N} \sum_{n=0}^{N-1} \operatorname{Sin}\left(\Omega_{0} k n\right) \\
\sigma_{I_{k}}^{2}=\sigma_{\xi}^{2} \frac{1}{N^{2}} \sum_{n=0}^{N-1} \operatorname{Sin}^{2}\left(\Omega_{0} k n\right)
\end{gathered}
$$

Proof:

The discrete Fourier transform of a discrete time signal $\xi_{k}$ is given by

$$
\xi_{k}=\frac{1}{N} \sum_{n=0}^{N-1} \xi(n) e^{-j \Omega_{0} k n}
$$

where $\Omega_{0}=\frac{2 \pi}{N}$ is the fundamental frequency. Decomposing the equation (5) into its real part and its imaginary part gives

$$
\begin{aligned}
\xi_{k} & =\frac{1}{N} \sum_{n=0}^{N-1} \xi(n) e^{-j \Omega_{0} k n} \\
& =\frac{1}{N} \sum_{n=0}^{N-1} \xi(n)\left(\operatorname{Cos}\left(\Omega_{0} k n\right)-j \operatorname{Sin}\left(\Omega_{0} k n\right)\right) \\
& =\frac{1}{N} \sum_{n=0}^{N-1} \xi(n) \operatorname{Cos}\left(\Omega_{0} k n\right)-j \frac{1}{N} \sum_{n=0}^{N-1} \xi(n) \operatorname{Sin}\left(\Omega_{0} k n\right) \\
& =R_{k}-j I_{k}
\end{aligned}
$$

where the second equality comes from Euler's identity. Hence the real and imaginary parts are linear combinations of normally distributed random variables

$$
\begin{aligned}
& R_{k}=\frac{1}{N} \sum_{n=0}^{N-1} \xi(n) \operatorname{Cos}\left(\Omega_{0} k n\right) \\
& I_{k}=\frac{1}{N} \sum_{n=0}^{N-1} \xi(n) \operatorname{Sin}\left(\Omega_{0} k n\right)
\end{aligned}
$$

As a result, it follows that $R_{k}$ and $I_{k}$ are also normally distributed random variables. The distribution parameters for the stochastic variable $R_{k}$ are calculated as follows (see, for example, (Box et al., 1978), page 87)

$$
\begin{gathered}
\mu_{R_{k}}=\mu_{\xi} \frac{1}{N} \sum_{n=0}^{N-1} \operatorname{Cos}\left(\Omega_{0} k n\right) \\
\sigma_{R_{k}}^{2}=\sigma_{\xi}^{2} \frac{1}{N^{2}} \sum_{n=0}^{N-1} \operatorname{Cos}^{2}\left(\Omega_{0} k n\right)
\end{gathered}
$$

The same reasoning is applicable to the imaginary part $I_{k}$. 
Remark 1

$\mu_{R_{k}}$ is equal to zero for $k \in\{1,2, \ldots, N-1\}$ and $\mu_{R_{0}}$ equals the mean value of the residual (i.e. $\left.\mu_{R_{0}}=\mu_{\xi}\right) . \mu_{I_{k}}$ is always equal to zero for $k \in\{0,1,2, \ldots, N-1\}$.

\section{Theorem 2}

The normalized squared gain $M_{k}^{2}$ defined as

$$
M_{k}^{2}=\left(\frac{R_{k}-\mu_{R_{k}}}{\sigma_{R_{k}}}\right)^{2}+\left(\frac{I_{k}-\mu_{I_{k}}}{\sigma_{I_{k}}}\right)^{2}
$$

has a $\chi^{2}$ distribution of 2 degrees of freedom.

Proof:

By definition the sum of $r$ independent squared random normal variables $N(0,1)$ has a $\chi^{2}$ distribution of $r$ degrees of freedom. Due to the normalization of $R_{k}$ and $I_{k}$, it follows that $\frac{R_{k}-\mu_{R_{k}}}{\sigma_{R_{k}}} \in N(0,1)$ and $\frac{I_{k}-\mu_{I_{k}}}{\sigma_{I_{k}}} \in N(0,1)$.

\subsection{Procedure}

The frequency domain model validation procedure is as follows

1. Calculate the residual as the difference of the real output and the model estimated output $(\xi(n)=y(n)-\hat{y}(n))$.

2. Calculate the discrete Fourier transform of the residual $\xi_{k}$.

3. Decompose each frequency component on its real part and imaginary part $\xi_{k}=R_{k}+j I_{k}$.

4. Calculate distribution parameters of the Real $\left(R_{k}\right)$ and Imaginary $\left(I_{k}\right)$ part for each frequency component $k$, as shown in Theorem 1 , that is:

- $\quad$ Real part $\left(R_{k}\right)$ : Calculate $\mu_{R_{0}}$ and $\sigma_{R_{k}}^{2}$ for $k \in\{0,1,2, \ldots, N-1\}$ (Equations (1) and (2)).

- Imaginary part $\left(I_{k}\right)$ : Calculate $\sigma_{I_{k}}^{2}$, for $k \in\{0,1,2, \ldots, N-1\}$ (Equations (3) and (4)).

5. Calculate the normalized magnitude spectrum for each frequency $k \in\{0,1,2, \ldots, N-1\}$ as follows:

$$
M_{k}^{2}=\left(\frac{R_{k}-\mu_{R_{k}}}{\sigma_{R_{k}}}\right)^{2}+\left(\frac{I_{k}-\mu_{I_{k}}}{\sigma_{I_{k}}}\right)^{2}
$$

6. Perform a hypothesis tests over each one of the normalized magnitude spectrum $M_{k}^{2}$, indexed by $k \in\{0,1,2, \ldots, N-1\}$, as follows:

- $H_{0}: M_{k}^{2} \in \chi_{2}^{2}$

- $H_{1}: M_{k}^{2} \notin \chi_{2}^{2}$ 
See next section for a deeper discussion on the hypothesis test procedure.
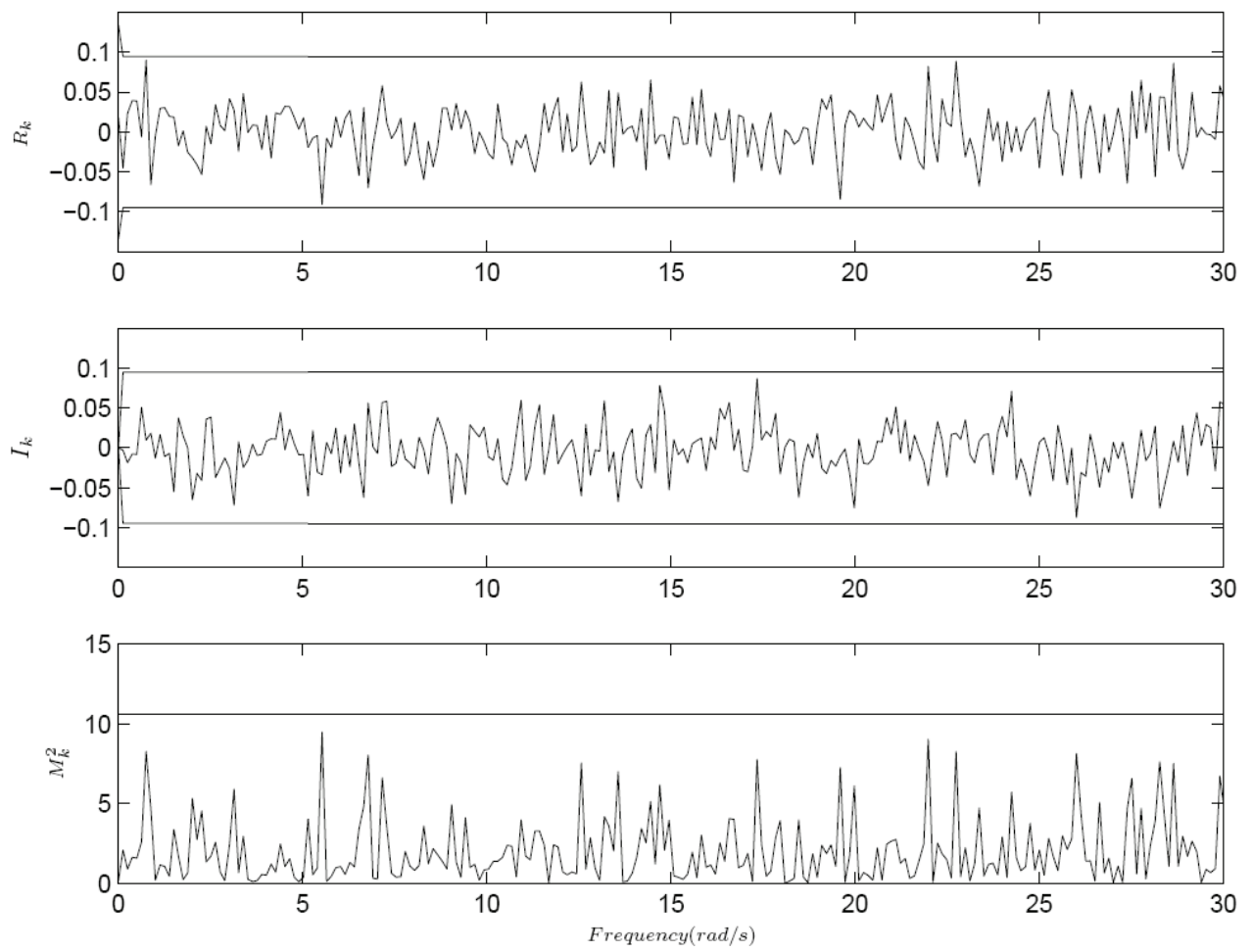

Figure 1. White noise example

The above steps are materialized in the following example. A realization of a normally distributed random variable of zero mean and unity variance is performed with 500 samples. The discrete Fourier transform of the realization is calculated and decomposed into its real and imaginary parts (i.e. $R_{k}, I_{k}$ ). The values of $R_{k}$ and $I_{k}$ are shown in figure 1 . They follow a normal variable distribution with parameters given by equations (1), (2), (3) and (4) (i.e. $R_{k} \in N\left(\mu_{R_{k}}=0, \sigma_{R_{k}}^{2}=0.001\right)$ and $I_{k} \in N\left(\mu_{I_{k}}=0, \sigma_{I_{k}}^{2}=0.001\right)$. On figure 1 the realizations of $R_{k}$ and $I_{k}$ are plotted together with the 3 sigma limits of their distribution (i.e. the $99.73 \%$ of the samples fall between the plotted limits). It can be seen that all points fall inside this range.

Finally the normalized magnitude spectrum is calculated following equation (6). The magnitude spectrum can be seen in figure 1 together with the $99.5 \%$ confidence limit of the $\chi_{2}^{2}$ distribution (i.e. the $99.5 \%$ of the samples fall between 0 and 10.6). All the magnitude frequency components remain below of the confidence limit so there are no reasons to invalidate the model. The whiteness test has passed. In the sequent section the hypothesis test features are described more thoroughly. 


\subsection{Hypothesis Test}

A hypothesis test is a statement about a random variable. This statement is expressed by means of two hypothesis $H_{0}$ and $H_{1} . H_{0}$ is called the null hypothesis and $H_{1}$ is the alternative hypothesis. In order to decide the validity of either the null hypothesis $H_{0}$ or the alternative hypothesis $H_{1}$, an estimation of a population parameter (e.g. mean or variance) is computed from a population sample and it is compared against the assumed population parameters. These population parameters are random variables too with certain mean and variance. If it is likely that the computed statistic is inside the population parameters distribution then $H_{0}$ is accepted, otherwise $H_{0}$ is rejected in favour of $H_{1}$. As a result, two errors are possible: to reject $H_{0}$ when it is true (type I error or $\alpha$ error), or to fail to reject $H_{0}$ when $H_{0}$ is false (type II error or $\beta$ error).

The hypothesis test is the last step of the presented procedure, where the decision of validation/invalidation of certain frequency component is taken. The hypothesis test to be applied in the proposed procedure is:

$$
\begin{aligned}
& H_{0}: M_{k}^{2} \in \chi_{2}^{2} \\
& H_{1}: M_{k}^{2} \notin \chi_{2}^{2}
\end{aligned}
$$

The hypothesis $H_{0}$ states that the normalized modulus $M_{k}^{2}$ of the $k$ frequency component is $\chi_{2}^{2}$ distributed. On the other hand the hypothesis $H_{1}$ states that the normalized modulus $M_{k}^{2}$ of the $k$ frequency component is not $\chi_{2}^{2}$ distributed.

\section{Remark 2}

The hypothesis test stated in (7) is applied to each frequency component, from $0 \mathrm{rad} / \mathrm{sec}$ up to the Nyquist frequency (i.e. $\frac{\pi}{T_{s}}$, where $T_{s}$ is the sample time).

In order to decide the validity of either the null hypothesis $H_{0}$ or the alternative hypothesis $H_{1}, M_{k}^{2}$ is computed. If it is "likely" that the value of $M_{k}^{2}$ lies inside the $\chi_{2}^{2}$ distribution then $H_{0}$ is accepted, otherwise $H_{0}$ is rejected in favour of $H_{1}$. As a result, two errors are possible as discussed above, to reject $H_{0}$ when it is true, or to fail to reject $H_{0}$ when $H_{0}$ is false.

The probability of rejecting $H_{0}$ when it is true is set by the user by choosing the confidence limit. For example, if the confidence limit is chosen to be 10.6 then the $99.5 \%$ of samples of a $\chi_{2}^{2}$ distribution fall inside the limit. This confidence limit sets the type I error. Following the example presented in the preceding section, the type I error was of $0.5 \%$, that is the $0.5 \%$ of the samples of a $\chi_{2}^{2}$ must be greater than 10.6. The type II error is more difficult to be calculated as it depends on knowledge of of the actual distribution followed by $M_{k}^{2}$.

The hypothesis test is then simply a check that any magnitude of the normalized spectrum is less than the test limit. If the value is greater then it is very unlikely and the model results invalidate for this frequency. 


\subsection{Open Loop Model Validation Example}

The proposed validation procedure in the frequency domain is applied to a stable plant in open loop (see figure 2). The real plant $G$ and the model $\hat{G}$ chosen to approximate it are:

$$
\begin{aligned}
& G=\frac{10}{(s+1)(s+10)} \\
& \hat{G}=\frac{1}{s+1}
\end{aligned}
$$

The Bode diagram comparing the real plant with the model is shown in figure 3.

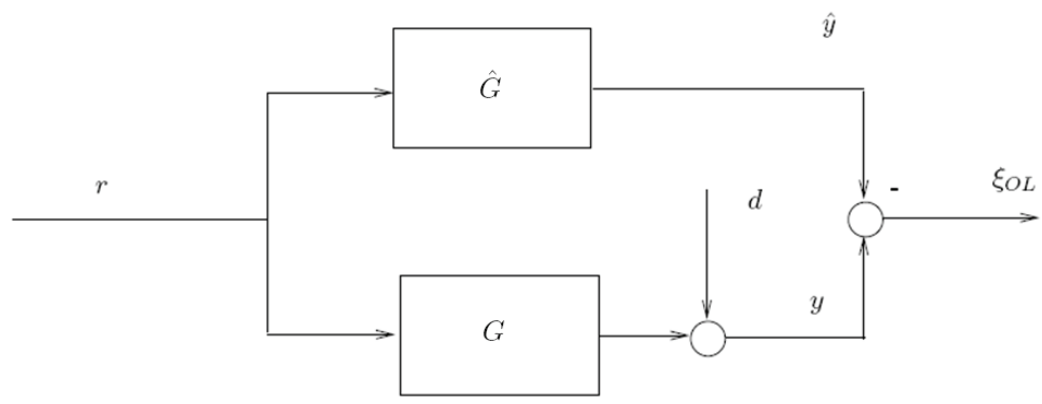

Figure 2. Open loop structure

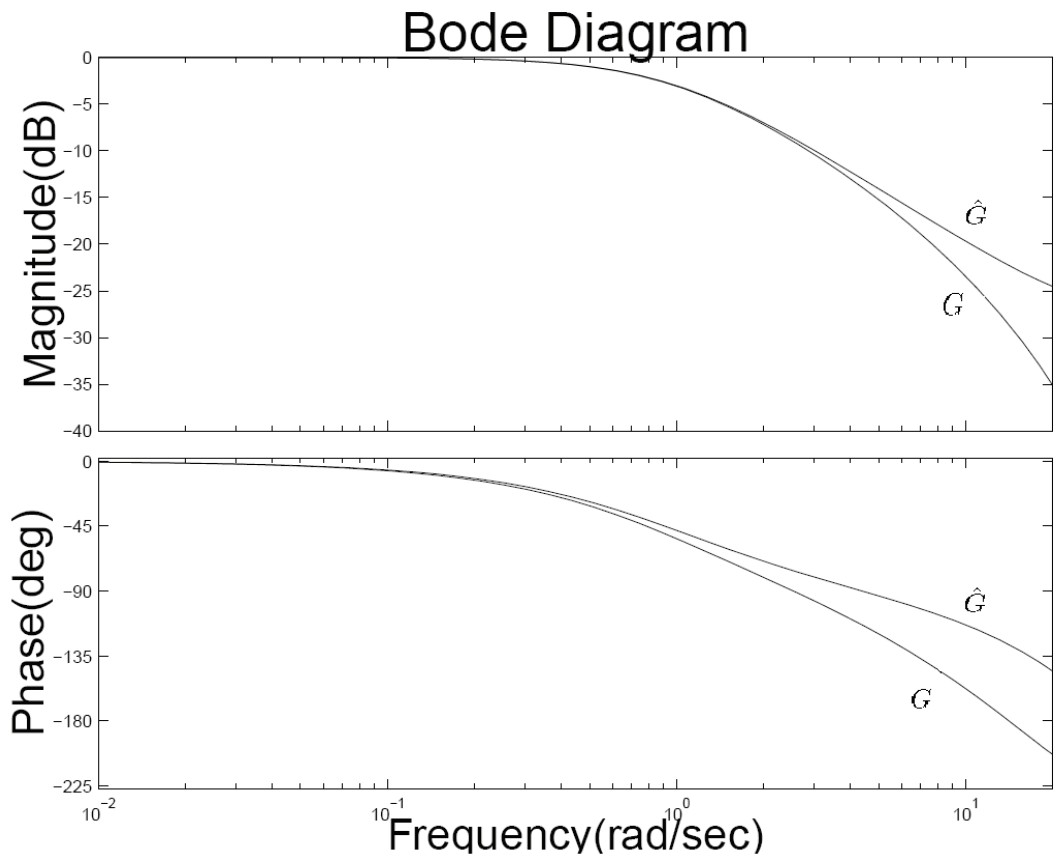

Figure 3. Comparison of Bode Diagram of plant and model 
The experimental setup is as follows. The residuals generated by the open loop structure of figure 2 are given by:

$$
\xi_{O L}=d+(G-\hat{G}) r
$$

Hence the residual $\xi_{O L}$ is just the noise $d$ if the model and the plant are equal (i.e. $\hat{G}=G$ ). Hence the residual has the same stochastic properties than the noise. The perturbation $d$ is assumed to be white noise with $\sigma=1$. The reference input $r$ is a train of sinusoids up to frequency $3 \mathrm{rad} / \mathrm{sec}$.

The validation procedure results can be seen in figure 4 . The model $\hat{G}$ shows no invalidation signs up to $1.4 \mathrm{rad} / \mathrm{sec}$. However for higher frequencies the hypothesis test fails to validate the model. As a conclusion we can state that, for the input applied, the model is correct for frequencies below to $1.4 \mathrm{rad} / \mathrm{sec}$. It is worth to mention that although between $1.7 \mathrm{rad} / \mathrm{sec}$ and $2.3 \mathrm{rad} / \mathrm{sec}$ there are no spikes out of the confidence limits, a deeper examination reveals that several consecutive spikes are abnormally high to belong a $\chi_{2}^{2}$ distribution. In order to detect this situation further probabilities should be checked (e.g. the probability that two consecutive points of a $\chi_{2}^{2}$ distribution be higher than some given value.)

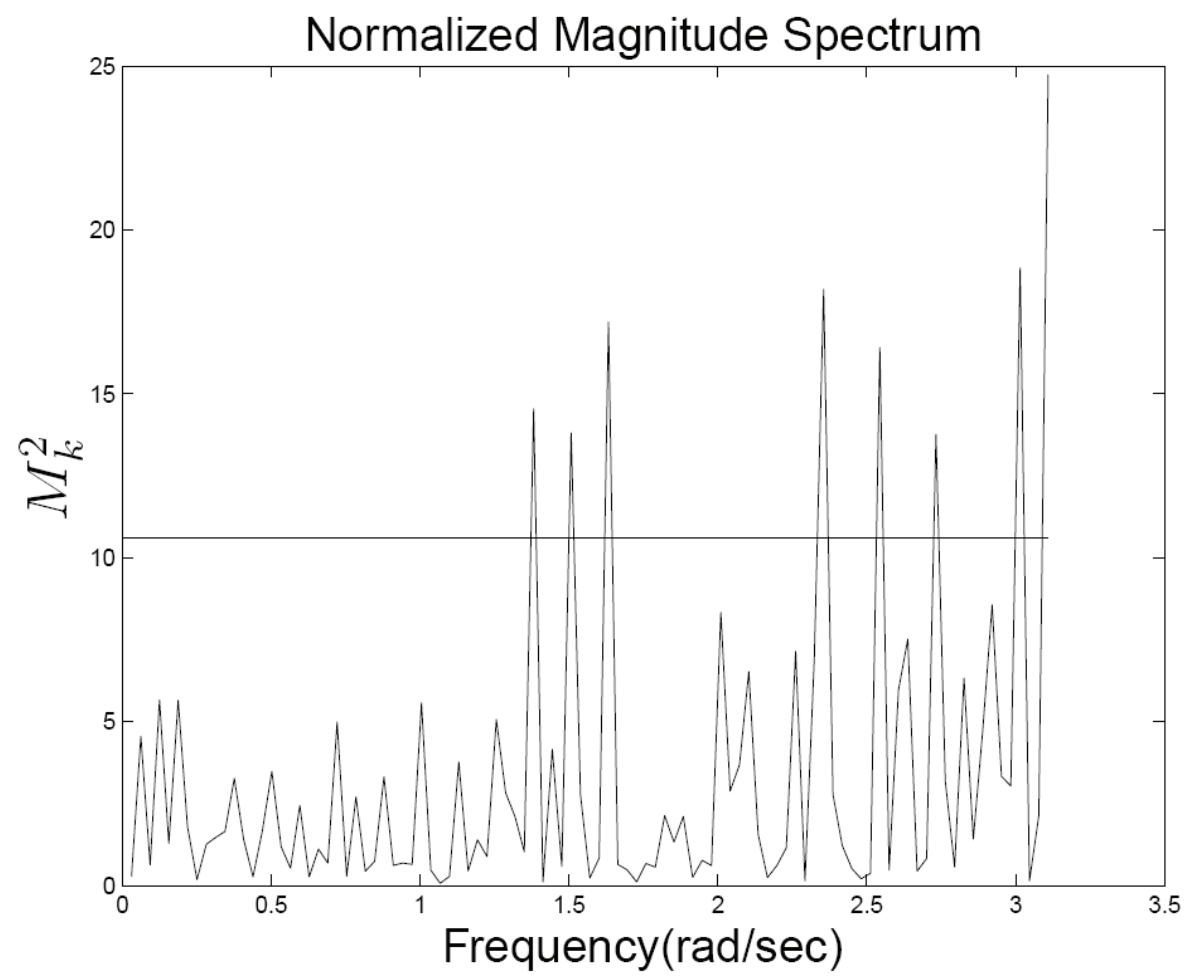

Figure 4. Normalized Magnitude Spectrum and Confidence Limit 
As a conclusion, the model $\hat{G}$ can be accepted as a good approximation of the plant $G$ up to frequency $1.4 \mathrm{rad} / \mathrm{sec}$. For higher frequencies the mismatch between model and plant is present up to the input bandwidth (i.e. $3 \mathrm{rad} / \mathrm{sec}$ ). It should be mention that this result is input dependent. However the results obtained up to now can serve as a guideline to design new input signals with suitable frequency contents for new identification steps (e.g. high energy around the frequencies were a significant error exists, that is between $1.4 \mathrm{rad} / \mathrm{sec}$ and $3 \mathrm{rad} / \mathrm{sec})$.

\section{Control Oriented Model Validation}

Model validation theory is aimed towards checking the model usefulness for some intended use. Thus the model validation procedure should take into account the model use, for example control design or prediction purposes. It is recognized in (Skelton, 1989) that arbitrary small model errors in open loop can lead to bad closed loop performance. On the other hand large open loop modelling errors do not necessarily lead to bad closed loop performance. As a result the model accuracy should be checked in such a way that the intended model use is taken into account in the model validation procedure.

An important aspect in the validation procedure to take into account is the intended model use and the validation conditions. In fact validation from open loop data can provide a different result that validation with closed loop data. Furthermore it is completely different to validate an open loop model than to compare two closed loops, the one with the model and the real one (See for example (Gevers et al., 1999)). This result points out the importance of the information that is being validated.

In order to consider the model intended use in the validation procedure, the conditions for data generation must be considered. In the following subsections different structures are proposed in order to compute the residuals and it is shown that they have considerable importance on the actual information that is validated. Its statistical properties are reviewed as the residuals must be statistically white under perfect model matching in order to apply the proposed algorithm. It is shown that the new model validation procedure introduced in this article can be endowed with the control oriented property by generating the residual using the structure presented in section 3.3.

\subsection{Open Loop Validation (Stable Plants)}

The model validation procedure is in open loop when there is no controller closing the loop. In that case, the structure used to validate the model is shown in figure 5. In open loop validation it is required that both, the plant $P$ and the plant model $\hat{P}$ be stable in order to obtain a bounded residual $\xi_{O L}$.

The residual $\xi_{O L}$ is given by the following expression:

$$
\xi_{O L}=d+(P-\hat{P}) r
$$

Now we analyze the residual characteristics when the model equals the plant and when there is a model plant mismatch. The residual $\xi_{O L}$ given by equation (8) is just the noise $d$ if the model and the plant are equal (i.e. $P=\hat{P}$ ). Hence the residual has the same stochastic properties than the noise. As a result, under white noise assumption, the residual $\xi_{O L}$ is also 
white noise and then will pass the frequency dependent validation procedure. On the other hand if there exist a discrepancy between the model and the plant, a new term $(P-\hat{P}) r$ appears in the residual. This term makes that the residual $\xi_{O L}$ is no longer white noise, hence the residual will not pass the frequency dependent test. It should be remarked however that the model-plant error which will be detected is deeply dependent on the reference signal $r$.

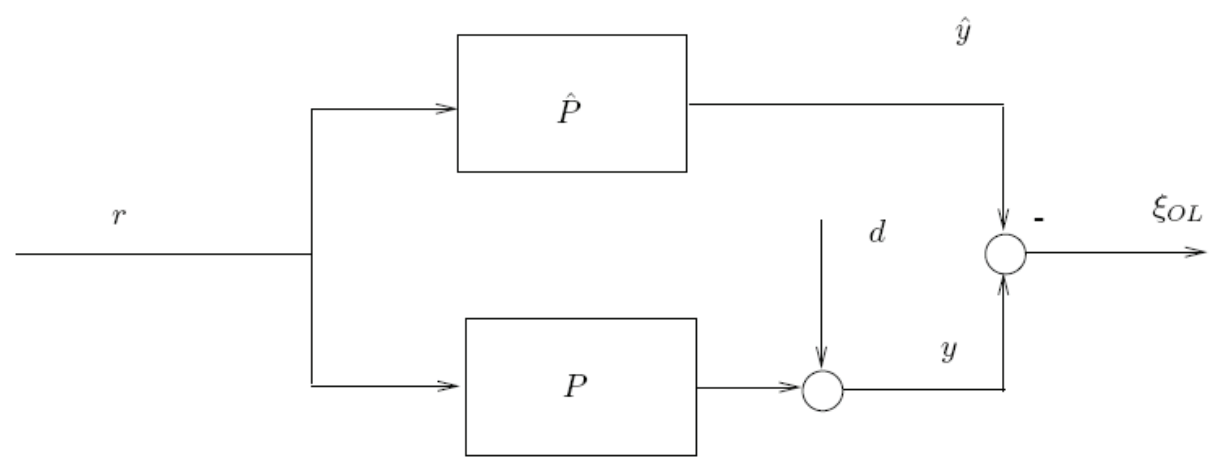

Figure 5. Open loop residual generation

\subsection{Closed Loop Validation (Unstable Plants)}

In the general closed loop validation case, the residual is generated as the comparison of two closed loops. On the one hand the closed loop formed by the controlled plant and on the other hand the closed loop formed by the controlled model (See figure 6). The main advantage of this configuration is that it permits validation of unstable models of unstable plants. Moreover, as we discuss below, the model-plant error is weighted.

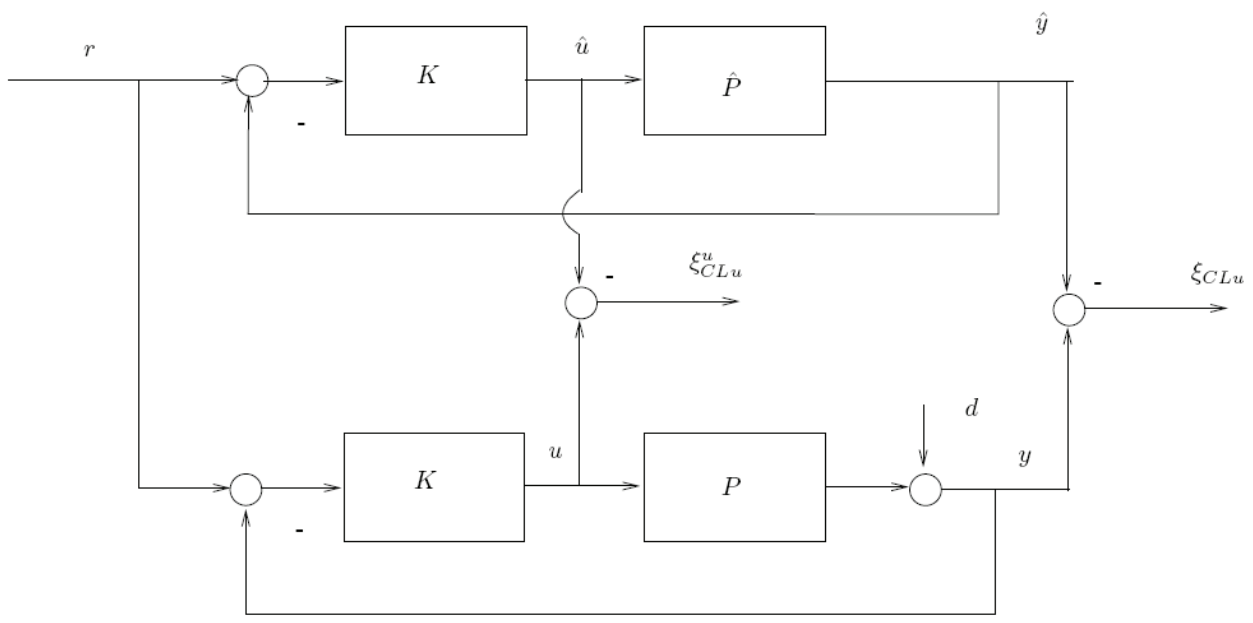

Figure 6. Closed loop residual generation (Unstable plants and models) 
The residual at the output $\xi_{C L u}$ (at the input $\xi_{C L u}^{u}$ ) of figure 6 is:

$$
\begin{aligned}
& \xi_{C L u}=S d+K S \hat{S}(P-\hat{P}) r \\
& \xi_{C L u}^{u}=-K S d+K K S \hat{S}(P-\hat{P}) r
\end{aligned}
$$

where $K$ is the controller, $S$ is the real sensitivity function (i.e. $S=(1+P K)^{-1}$ ) and $\hat{S}$ is the model sensitivity function (i.e. $\left.\hat{S}=(1+\hat{P} K)^{-1}\right)$. In the case there is a perfect model-plant match, that is when $\hat{P}=P$, the residual $\xi_{C L u}\left(\xi_{C L u}^{u}\right)$ yields $S d(-K S d)$. As a result, independently of the noise characteristics, the residual is always autocorrelated, as the noise is filtered by $S(-K S)$. Hence it is not possible to perform the frequency dependent whiteness test in order to validate the model.

If there is a model-plant mismatch (i.e. $\hat{P} \neq P$ ), a new term arises in residual $\xi_{C L u}\left(\xi_{C L u}^{u}\right)$. This term is $K S \hat{S}(P-\hat{P}) r(K K S \hat{S}(P-\hat{P}) r)$, that is the model plant error weighted by $K S \hat{S}$ ( $K K S \hat{S})$. As a result, the relative importance of the model plant error is weighted, in such a way that if the gain of term $K S \hat{S}$ ( $K K S \hat{S})$ is "low" the error is not important but when the term gain $K S \hat{S}$ ( $K K S \hat{S}$ ) is "high" then the error is amplified. Thus we can see how the closed loop validation takes into account the model errors for control design purposes.

Summing up, although the closed loop validation structure presented in figure 6 is control oriented and allows the validation of unstable models, the residual generated by this structure is not suited for performing the frequency dependent validation procedure. In the next section we present a structure that allows performing the frequency dependent model validation on residuals generated in a control oriented way.

\subsection{Closed Loop Validation (Stable Plants)}

In this section we present a structure for generating the residual in such a way that first, it is control oriented and secondly it is suitable for the frequency dependent control oriented procedure proposed. The structure is shown in figure 7.

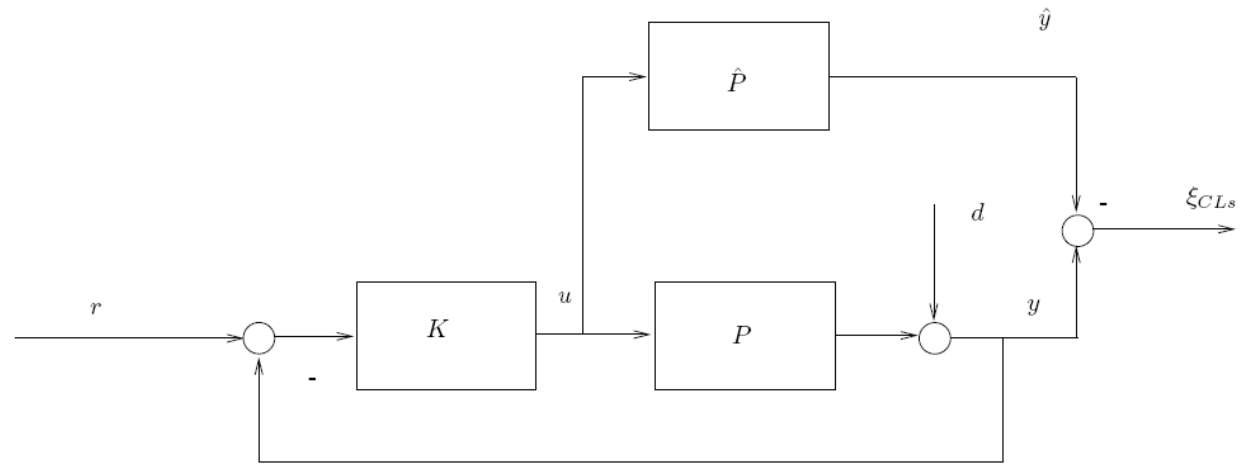

Figure 7. Closed loop residual generation (Stable models) 
In this case, the residual is given by:

$$
\xi_{C L S}=\frac{S}{\hat{S}} d+K S(P-\hat{P}) r
$$

where $K$ is the controller, $S$ is the real sensitivity function (i.e. $S=(1+P K)^{-1}$ ) and $\hat{S}$ is the model sensitivity function (i.e. $\left.\hat{S}=(1+\hat{P} K)^{-1}\right)$. The residual $\xi_{C L S}$ given by equation (10) is the noise $d$ filtered by the fraction of the real Sensitivity function $S=(1+P K)^{-1}$ and the Sensitivity function of the model $\hat{S}=(1+\hat{P} K)^{-1}$ plus a term that is the discrepancy of the plants weighted by the control sensitivity function (i.e. $K S$ ). If the model and the plant are equal (i.e. $\hat{P}=P$ ) then the real sensitivity function $S$ and the model sensitivity function $\hat{S}$ are equal so the first term of equation (10) yields the noise $d$. Moreover the second term, under the same perfect model-plant matching assumption, is zero. Hence in this case the residuals are again the noise $d$, thus it is suitable for our proposed frequency dependent validation algorithm.

On the other hand, if a discrepancy exists between the model $\hat{P}$ and the plant $P$, the division of $S$ by $\hat{S}$ is no longer unity but equals a transfer function resulting from the noise $d$ filtered by $S / \hat{S}$ (i.e. autocorrelated). Additionally the second term of equation (10) gives a signal proportional to the model-plant error weighted by the control sensitivity function (i.e. $K S$ ).

The presented structure is then suited to generate the residual in order to be used by the proposed validation algorithm.

\section{Application of the Frequency Dependent Model Validation to Iterative Identification and Control Schemes}

Iterative identification and control design schemes improve performance by designing new controllers on the basis of new identified models (Albertos and Sala, 2002). The procedure is as follows: an experiment is performed in closed loop with the current designed controller. A new model is identified with the experimental data and a new controller is designed using the new model. The procedure is repeated until satisfactory performance is achieved. The rationale behind iterative control is that if iteratively "better" models are identified, hence "better" performing controllers can be designed. However the meaning of "better" model needs some clarification. The idea of modelling the "true" plant has proven to be bogus (Hjalmarsson, 2005). Instead a good model for control is one that captures accurately the interesting frequency range for control purposes. In fact the model has no other use than to design a controller, thus the use of the model is instrumental (Lee et al., 1995). Hence, once a model is obtained it is necessary to validate it. On the iterative identification and control schemes this should be done each time a new model is identified (i.e. at each iteration).

The main problem of the validation methods reviewed is that the answer is a binary result (i.e. validated/invalidated). However models are neither good nor bad but have a certain valid frequency range (e.g. normally models are good at capturing low frequency behaviour 
but their accuracy degrades at higher frequencies). Moreover the iterative identification and control procedures have their own particular requirements

- Is it possible to improve an existing model? Is the data informative enough to attempt a new identification?

- How can the model be improved? Is the model order/structure rich enough to capture the interesting features of the plant?

- How authoritative can be the controller designed on the basis of the new model? Which is the validity frequency range of my model?

The above requirements for iterative control can not be provided by the classical model validation approaches above introduced because

- No indication on the possibility to improve an existing model. This problem is solved in (Lee et al., 1995) by the use of classical validation methods (i.e. cross-correlation test) together with the visual comparison of two power spectra.

- In iterative identification and control approaches a low order model is fitted to capture the frequency range of interest for control. Hence undermodelling is always present. This fact makes it difficult to apply traditional model validation schemes as the output of the validation procedure is a binary answer (i.e. validated/no validated) (Ljung, 1994).

- No indication on how to improve the model on the next iteration (i.e. model order selection and/or input experiment design).

- No indication on the model validity range for control design (i.e. controller bandwidth selection).

In the next section we present the benefits on the proposed validation algorithm on the iterative identification and control schemes.

\subsection{Model Validation on Iterative Identification and Control Schemes}

The benefits of the frequency dependent model validation for the iterative identification and control schemes hinge on the frequency domain information produced by the algorithm. It is possible to assess for what frequency range a new model should be identified (perhaps increasing the model order) and what frequency content should contain the input of the experiment. Moreover we have information over the frequency range for which the model is validated, thus it is possible to choose the proper controller bandwidth.

The benefits of the frequency dependent model validation approach over iterative identification and control (see figure 8 ) are:

- Designing the input experiment for the next identification step. It is well known that the identified model quality hinges on the experiment designed to obtain the data. The experiment should contain high energy components on the frequency range where the model is being validated if informative data are pursued for a new identification in the following step.

- $\quad$ Detecting model undermodelling and/or choosing model order. A higher order model can be fitted over the frequency range where the current model is being invalidated. It can be done even inside the current iteration step without the need of performing a new experiment. In (Balaguer et al., 2006c) a methodology to add poles and zeroes to an existing model can be found.

- Selecting controller bandwidth on the controller design step. Once a frequency range of the model has been validated, if no further improvement of the model is sought, the final controller designed should respect the allowable bandwidths of the model. 
These issues are shown by means of the next section illustrative example.

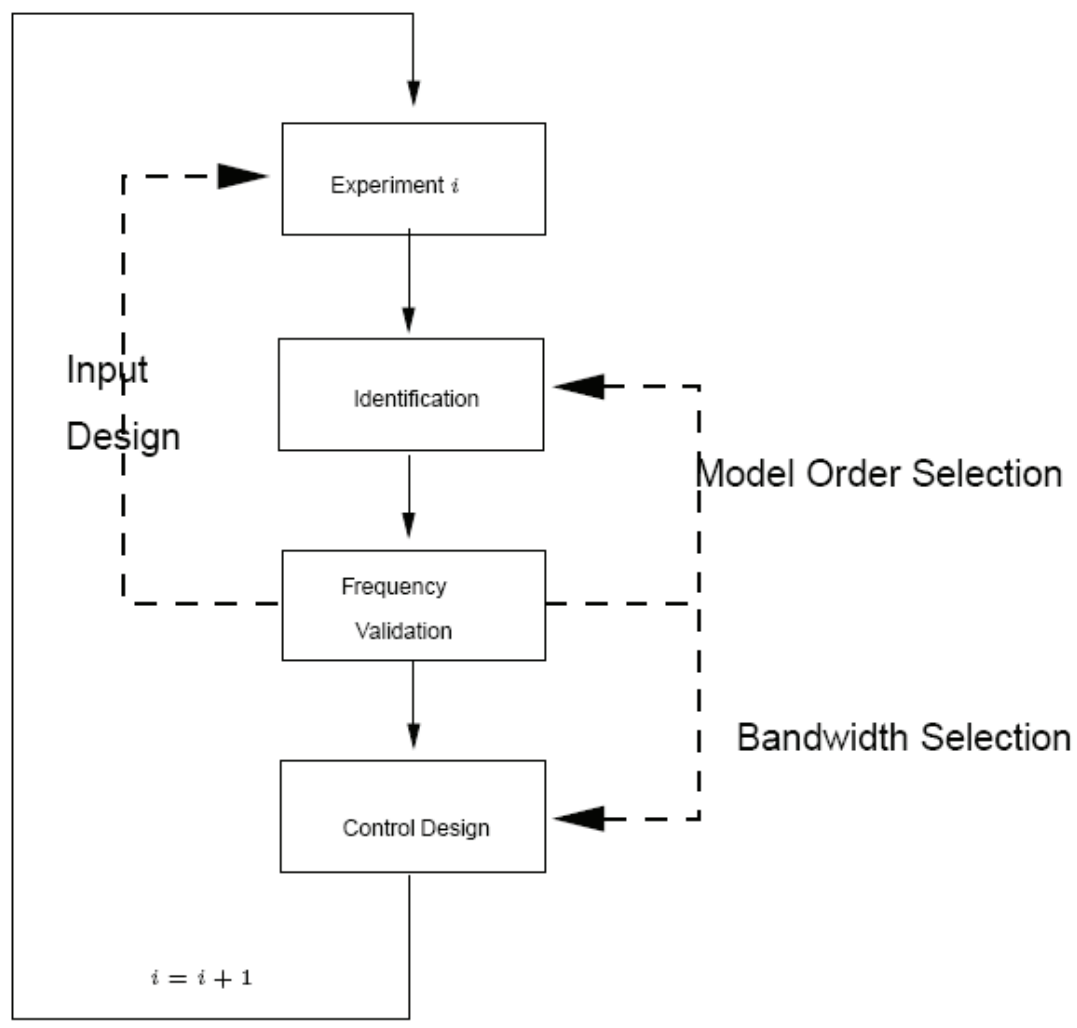

Figure 8. Frequency dependent model validation on iterative control

\subsection{Illustrative Example}

The present example is the application of the proposed frequency domain model validation to an iterative identification and control design. As baseline we take the Iterative Control Design example presented in (Albertos and Sala, 2002), page 126, where a stable plant with highfrequency resonant modes is controlled by successive plant identification (e.g. step response) and the subsequent controller design (e.g. model matching and cancellation controller). We apply to the successive models and controllers given in the example our frequency domain model validation procedure. Moreover we propose a customized structure in order to generate adequate residuals to claim for a control oriented model validation.

The proposed structure to generate the residuals is in closed loop, as shown in figure 7. The residual is given by equation (10), which is repeated here, following the example notation, for the sake of clarity:

$$
\xi_{C L s}=\frac{S}{\hat{S}} d+K S(G-\hat{G}) r
$$


The experimental setup is as follows. First a model of the plant $\hat{G}$ is obtained by a step response identification. For this model successive controllers $K$ are designed by imposing more stringent reference models $M$. When the closed loop step response is unsatisfactory, a new model is identified and the controller design steps repeated. The measurement noise $d$ is white noise with $\sigma=10^{-2}$. The reference input $r$ is a train of sinusoids up to frequency $200 \mathrm{rad} / \mathrm{sec}$. Finally, the plant $G$ to be controlled is sixth order, given by

$$
G=\frac{10^{6}(s+1000)}{\left(s^{2}+0.002 s+1000^{2}\right)\left(s^{2}+0.1 s+50^{2}\right)(s+0.1)(s+0.2)}
$$
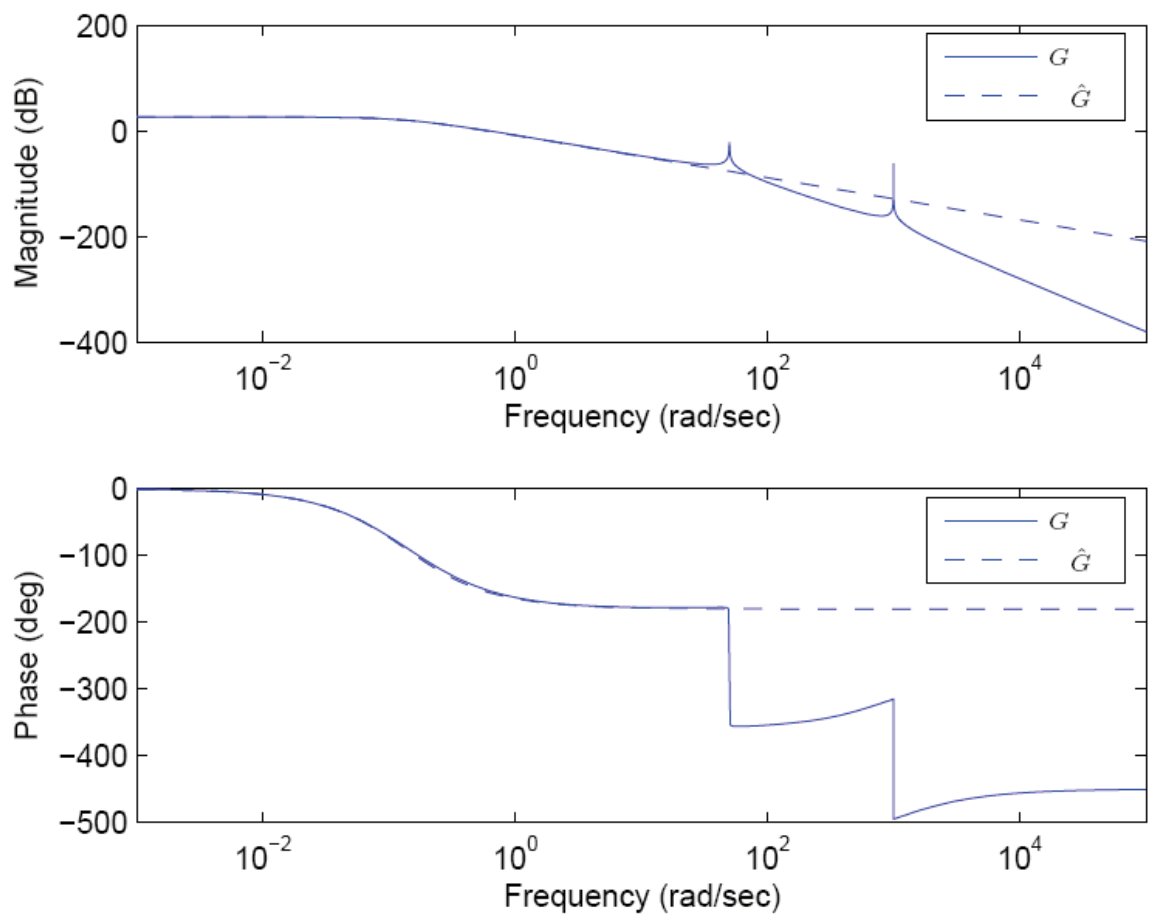

Figure 9. Bode diagrams of the plant and the model

\section{First Iteration}

The first identified model $\hat{G}_{0}$ and the model reference $M_{01}$ used for controller design are:

$$
\begin{aligned}
& \hat{G}_{0}=\frac{20}{(1+7.4 s)^{2}} \\
& M_{01}=\frac{0.5^{2}}{(s+0.5)^{2}}
\end{aligned}
$$


The bode plot of the real plant $G$ and the first model $\hat{G}_{0}$ are shown in figure 9. The frequency domain validation is applied, given a positive validation result, as can be seen in the first plot of figure 10 .
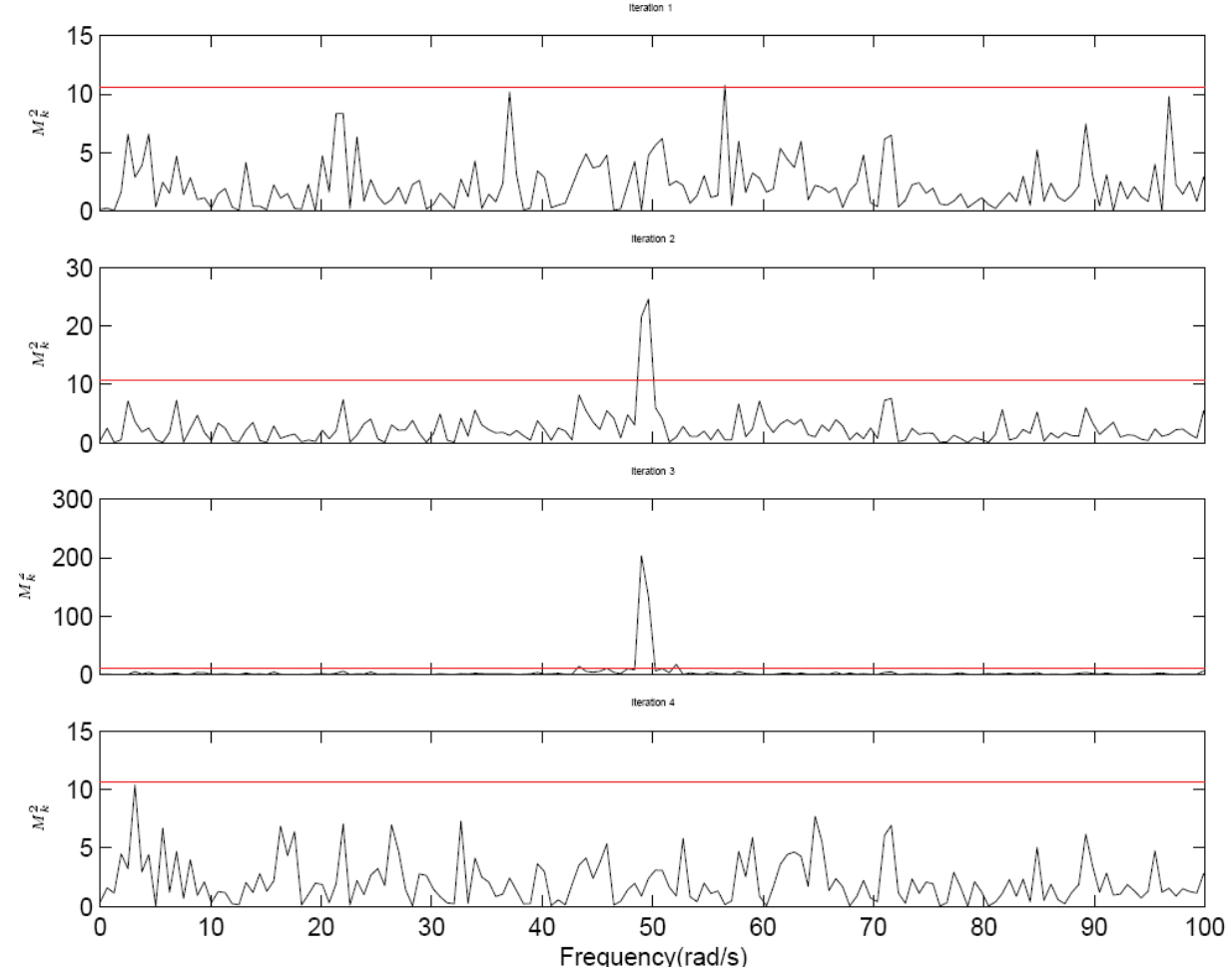

Figure 10. Frequency dependent validation result at each iteration

\section{Second Iteration}

Following the positive validation result of the first iteration the same model is kept as valid and the performance is pushed forward by a new, more stringent, reference model $M_{02}$ :

$$
\begin{aligned}
& \hat{G}_{0}=\frac{20}{(1+7.4 s)^{2}} \\
& M_{02}=\frac{3^{2}}{(s+3)^{2}}
\end{aligned}
$$

The validation test invalidate the model for frequencies around $50 \mathrm{rad} / \mathrm{sec}$ (see plot 2 of figure 10. This is due to the non modelled resonance peak as can be seen in the bode diagram of figure 9.

\section{Third Iteration}

In (Albertos and Sala, 2002), the new identification step is taken after pushing even forward the desired reference model $M_{03}$ : 


$$
\begin{aligned}
& \hat{G}_{0}=\frac{20}{(1+7.4 s)^{2}} \\
& M_{03}=\frac{5^{2}}{(s+5)^{2}}
\end{aligned}
$$

The invalidation of the model for frequencies around $50 \mathrm{rad} / \mathrm{sec}$ for this controller is evident (plot 3 of figure 10).

\section{Fourth Iteration}

In (Albertos and Sala, 2002) a new model plant is identified due to the unacceptable closed loop behaviour for the controller designed with the reference model $M_{03}$. The new identified plant $G_{1}$ captures the first resonance peak of the plant. The reference model is $M_{11}$ which keeps the same time constant as the former reference model $M_{03}$.

$$
\begin{aligned}
& \hat{G}_{1}=\hat{G}_{\cdot 0} \frac{0.01^{2}+50^{2}}{(s+0.01+50 j)(s+0.01-50 j)} \\
& M_{11}=\frac{5^{4}}{(s+5)^{4}}
\end{aligned}
$$

The model validation result shows that now, the model is validated for all the frequency range covered by the input (plot 4 of figure 10).

Summarizing the example results, we have shown how the frequency dependent model validation scheme can be helpful to guide the identification step by aiming towards the interesting frequencies content that an identification experiment should excite. The procedure is also helpful to choose the appropriate controller bandwidth suitable for the actual model accuracy. Moreover it has been proven that the proposed methodology can be applied in iterative identification and control design schemes and the validation can be control oriented.

\section{Conclusion}

In this paper a new algorithm for model validation has been presented. The originality of the approach is that it validates the model in the frequency domain rather than in the time domain. The procedure of validating a model in the frequency domain has proven to be more informative for control identification and design purposes than classical validation methods.

- Firstly, the model is neither validated nor invalidated. Instead valid/invalid frequency ranges are given.

- Secondly, the invalidated frequency range is useful in order to determine the new experiment to identified better models in those frequency ranges.

- Thirdly, the model validity frequency range establishes a maximum controller bandwidth allowable for the model quality.

Our model validation procedure is of interest for Iterative Identification and Control schemes. Normally these schemes start with a low quality model and low authoritative controller which are improved iteratively. As a result poor models must be improved. This 
raises the questions on model validation and controller bandwidth that our approach helps to solve. Classical validation methods would invalidate the first low quality model meanwhile it is of use for future improvements.

Another application area of the proposed frequency dependent model validation is the tuning and validation of controllers. In this way it is possible to find low order controllers that behave similarly to high order ones in some frequency band.

Summing up the major advantage of the proposed algorithm is the frequency viewpoint which enables a richer validation result than the binary answer of the existing algorithms.

\section{References}

Albertos, P. \& Sala, A. (2002). Iterative Identification and Control, Springer

Balaguer, P. \& Vilanova, R. (2006a). Model Validation on Iterative Identification and Control Schemes, Proceedings of $7^{\text {th }}$ Portuguese Conference on Automatic Control, pp. 14-17, Lisbon

Balaguer, P. \& Vilanova, R. (2006b). Quality assessment of models for iterative/adaptive control, Proceedings of the $45^{\text {th }}$ Conference on Decision and Control, pp. 14-17, San Diego

Balaguer, P., Vilanova, R \& A. Ibeas. (2006c). Validation and improvement of models in the frequency domain, Computational Engineering in System Applications, pp. 14-17, Beijing

Balaguer, P., Wahab, N.A., Katebi, R. \& Vilanova, R. (2008). Multivariable PID control tuning: a controller validation approach, Emerging Technologies and Factory Automation, pp. 14-17, Hamburg

Box, G., Hunter W. \& Hunter, J. (1978). Statistics for Experimenters. An Introduction to Design, Data Analisis and Model Building, John Wiley E Sons, Inc.

Chen, J. \& Gu, G. (2000). Control Oriented System Identification. An $H_{\infty}$ Approach, John Wiley $\mathcal{E}$ Sons, Inc.

Gevers, M.; Codrons, B. \& Bruyne, F. (1999). Model Validation in Closed Loop, Proceedings of the American Control Conference

Hjalmarsson, H. (2005). From Experiment Design to Closed-Loop Control. Automatica, Vol. 41, page numbers (393-438)

Lee, W., Anderson, B., Mareels, I. and Kosut, R. (1995). On Some Key Issues in the Windsurfer Approach to Adaptive Robust Control. Automatica, Vol. 31, page numbers (1619-1636)

Ljung, L. (1994). System Identification. Theory for the User, Prentice-Hall

Skelton, R. (1989). Model Error Concepts in Control Design. International Journal of Control, Vol. 49, No. 5, page numbers (1725-1753)

Soderstrom, T. \& Stoica, P. (1989). System Identification, Prentice Hall International Series in Systems and Control Engineering. 


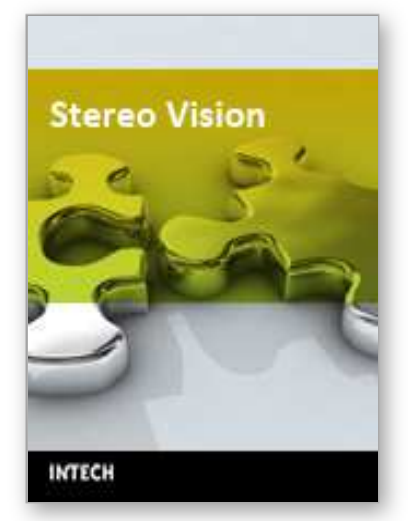

\author{
Frontiers in Adaptive Control \\ Edited by Shuang Cong
}

ISBN 978-953-7619-43-5

Hard cover, 334 pages

Publisher InTech

Published online 01, January, 2009

Published in print edition January, 2009

The objective of this book is to provide an up-to-date and state-of-the-art coverage of diverse aspects related to adaptive control theory, methodologies and applications. These include various robust techniques, performance enhancement techniques, techniques with less a-priori knowledge, nonlinear adaptive control techniques and intelligent adaptive techniques. There are several themes in this book which instance both the maturity and the novelty of the general adaptive control. Each chapter is introduced by a brief preamble providing the background and objectives of subject matter. The experiment results are presented in considerable detail in order to facilitate the comprehension of the theoretical development, as well as to increase sensitivity of applications in practical problems

\title{
How to reference
}

In order to correctly reference this scholarly work, feel free to copy and paste the following:

Pedro Balaguer and Ramon Vilanova (2009). A New Frequency Dependent Approach to Model Validation, Frontiers in Adaptive Control, Shuang Cong (Ed.), ISBN: 978-953-7619-43-5, InTech, Available from: http://www.intechopen.com/books/frontiers_in_adaptive_control/a_new_frequency_dependent_approach_to_ model_validation

\section{INTECH}

open science | open minds

\section{InTech Europe}

University Campus STeP Ri Slavka Krautzeka 83/A 51000 Rijeka, Croatia Phone: +385 (51) 770447 Fax: +385 (51) 686166 www.intechopen.com

\author{
InTech China \\ Unit 405, Office Block, Hotel Equatorial Shanghai \\ No.65, Yan An Road (West), Shanghai, 200040, China \\ 中国上海市延安西路65号上海国际贵都大饭店办公楼405单元 \\ Phone: +86-21-62489820 \\ Fax: +86-21-62489821
}


(C) 2009 The Author(s). Licensee IntechOpen. This chapter is distributed under the terms of the Creative Commons Attribution-NonCommercialShareAlike-3.0 License, which permits use, distribution and reproduction for non-commercial purposes, provided the original is properly cited and derivative works building on this content are distributed under the same license. 